\title{
New Exact Solutions of Rational Expansion method for the Variable Coefficient Nonlinear Equation with Forced Term
}

\author{
http://dx.doi.org/10.3991/ijoe.v9iS4.2663 \\ Juan Liu, Zhan-wei Hou \\ Henan Polytechnic University, Jiaozuo, P R China
}

\begin{abstract}
With the aid of symbolic computation system Maple, several new kinds of generalized exact solutions for the variable coefficient combined $\mathrm{KdV}$ equation and Chaffee-Infante equation with forced term are obtained by using a new generalize Riccati equation rational expansion method. This approach can also be applied to other variable coefficient nonlinear evolution equations.
\end{abstract}

Index Terms-forced term, generalized Riccati equation rational expansion method, solitary-wave-like solutions, variable coefficient combined $\mathrm{KdV}$ equation.

\section{INTRODUCTION}

In the nonlinear science, many important phenomena in various fields can be described by the nonlinear evolution equations (NLEEs). Searching for exact solutions of NLEEs plays an important and significant role in the study on the dynamics of those phenomena[1-14]. Recently, much attention has been paid to the variable-coefficient nonlinear equations which can describe many nonlinear phenomena more realistically than their constantcoefficient ones. Many powerful methods are been presented to obtain the exact solutions of nonlinear evolution equation, such as Variational method, truncation expansion method, the homogeneous balance method, Bäcklund transformation method, F - expansion method, the method of separation of variables, Jacobi elliptic function method, deformation mapping method and so on[8-14].

In this paper, by use of the generalized Riccati equation, we propose a new algebraic method to construct some new exact solutions of the $\mathrm{KdV}$ equation and ChaffeeInfante equation with variable coefficients. Including many kinds of solitary-wave-like solutions and likeperiodical solutions, many solutions are new.

The rest of paper is arranged as follows. In section 2, we briefly describe our method--the new Riccati equation rational expansion method. In section 3, we apply the new method to the KdV equation and Chaffee-Infante equation with variable coefficients. Finally, in section 4, some conclusions are given.

\section{SUMMARY OF THE GENERALIZED RICCATI EQUATION RATIONAL EXPANSION METHOD}

In the following we would like to outline the main content of our method.
For the given nonlinear evolution system with some physical fields $u_{i}(x, t)$ in two variables $x, t$,

$$
\nabla_{i}\left(u_{i}, u_{i t}, u_{i x}, u_{i t t}, u_{i x x}, u_{i x t} \Lambda\right)=0
$$

by using the wave transformation $u_{i}(x, t)=U_{i}(\xi)$,

$\xi=x+\lambda t$, where $\lambda$ is a constant to be determined later. Then the nonlinear partial differential (2) is reduced to a nonlinear ordinary differential equation (ODE):

$$
\Delta_{i}\left(U_{i}, U_{i}^{\prime}, U_{i}^{\prime \prime}, \Lambda\right)=0 \text {, }
$$

where ' $=\frac{d}{d \xi}$.

We introduce a new ansäta in terms of finite rational formal expansion in the following forms:

$$
U_{i}=a_{i 0}+\sum_{j=1}^{m_{i}} \frac{a_{i j} \psi^{j}(\xi)}{(1+\mu \psi(\xi))^{j}}
$$

where

$$
a_{i 0}=a_{i 0}(t), a_{i j}=a_{i j}(t)\left(i=1,2, \Lambda ; j=1,2, \Lambda, m_{i}\right)
$$

are functions of $t$ to be determined later. $\xi=\xi(x, t)$ are arbitrary functions with $x$ and $t, \mu$ are constants to be determined later. The parameter $m_{i}$ can be found by balancing the highest order derivative term and the nonlinear terms in (1) or (2):

(i) If $m_{i}$ is a positive integer then go on ;

(ii) If $m_{i}$ is a fraction or a negative integer, we make the transformation $u(\xi)=v^{m_{i}}(\xi)$ then determine $m_{i}$ again.

Where the new variable $\psi=\psi(\xi)$ satisfies the generalized Riccati equation as following:

$$
\psi^{\prime}-\left(h_{1}+h_{2} \psi^{2}\right)=\frac{d \psi}{d \xi}-\left(h_{1}+h_{2} \psi^{2}\right)=0,
$$

where “' " $=\frac{d}{d \xi}, h_{1}, h_{2}$ are arbitrary real numbers, behind the same.

Substitute (3) into (4) along with (2), then set all coefficients of $\psi^{i}(\xi)(i=1,2, \Lambda)$ of the resulting system's numerator to be zero to get an over-determined system of nonlinear algebraic equations with respect to $k(t), l(t), a_{i 0}(t), a_{i j}(t)\left(i=1,2, \Lambda ; j=1,2, \Lambda m_{i}\right)$ 
and $\mu$. Solving the over-determined system of nonlinear algebraic equations by use of Maple, we would end up with the explicit expressions for $k(t), l(t), a_{i 0}(t), a_{i j}(t)$ ( $\left.i=1,2, \Lambda ; j=1,2, \Lambda m_{i}\right)$ and $\mu$.

With the aid of Maple, we obtain the general solutions of (4) which are now listed as following:

1) when $h_{1} h_{2}>0$

$$
\begin{aligned}
& \psi(\xi)=\frac{\sqrt{h_{1} h_{2}}}{h_{2}} \tan \left(\sqrt{h_{1} h_{2}} \xi+c_{1}\right), \\
& \psi(\xi)=\frac{\sqrt{h_{1} h_{2}}}{h_{2}} \cot \left(\sqrt{h_{1} h_{2}} \xi+c_{2}\right) .
\end{aligned}
$$

2) when $h_{1} h_{2}<0$

$$
\begin{aligned}
& \psi(\xi)=\frac{\sqrt{-h_{1} h_{2}}}{h_{2}} \tanh \left(\sqrt{-h_{1} h_{2}} \xi+c_{3}\right), \\
& \psi(\xi)=\frac{\sqrt{-h_{1} h_{2}}}{h_{2}} \operatorname{coth}\left(\sqrt{-h_{1} h_{2}} \xi+c_{4}\right) .
\end{aligned}
$$

3) when $h_{1}=0$ and $h_{2} \neq 0$

$$
\psi(\xi)=-\frac{1}{h_{2} \xi+c},
$$

where $\xi=k(t) x+l(t)$, and $c, c_{1}, c_{2} c_{3}, c_{4}$ are arbitrary constants.

Remark 1 As is well known, there exist the following relationship:

$$
\begin{gathered}
\cos (2 \xi)=2 \cos ^{2}(\xi)-1=1-2 \sin ^{2}(\xi), \\
\sin (2 \xi)=2 \sin (\xi) \cos (\xi), \\
\cosh (2 \xi)=2 \cosh ^{2}(\xi)-1=2 \sin ^{2}(\xi)+1, \\
\sinh (2 \xi)=2 \sinh (\xi) \cosh (\xi) .
\end{gathered}
$$

So (5) and (6) can be listed as:

$\psi(\xi)=\frac{\sqrt{h_{1} h_{2}}}{h_{2}}\left(\csc \left(\sqrt{4 h_{1} h_{2}} \xi+c_{5}\right) \pm \cot \left(\sqrt{4 h_{1} h_{2}} \xi+c_{5}\right)\right)$

$\psi(\xi)=\frac{\sqrt{h_{1} h_{2}}}{h_{2}}\left(\sec \left(\sqrt{4 h_{1} h_{2}} \xi+c_{6}\right) \pm \tan \left(\sqrt{4 h_{1} h_{2}} \xi+c_{6}\right)\right)$

where (7) and (8) can also be listed as:

$\psi(\xi)=\frac{\sqrt{-h_{1} h_{2}}}{h_{2}}\left(\operatorname{coth}\left(\sqrt{-4 h_{1} h_{2}} \xi+c_{7}\right) \pm \csc \left(\sqrt{-4 h_{1} h_{2}} \xi+c_{7}\right)\right)$ $\psi(\xi)=\frac{\sqrt{-h_{1} h_{2}}}{h_{2}}\left(\tanh \left(\sqrt{-4 h_{1} h_{2}} \xi+c_{8}\right) \pm i \operatorname{sech}\left(\sqrt{-4 h_{1} h_{2}} \xi+c_{8}\right)\right)$

Remark 2 By use of the Euler formula, (5) and (6) can also be listed as exp functions:

$$
\psi(\xi)=\frac{\sqrt{h_{1} h_{2}}}{h_{2}} \frac{\left(1-i c e^{2 i \xi \sqrt{h_{1} h_{2}}}\right)}{\left(1+c e^{2 i \xi \sqrt{h_{1} h_{2}}}\right)}
$$

while (7) and (8) be listed as:

$$
\left.\psi(\xi)=\frac{\sqrt{-h_{1} h_{2}}}{h_{2}} \frac{\left(1+c e^{2 \xi \sqrt{-h_{1} h_{2}}}\right.}{\left(1-c e^{2 \xi \sqrt{-h_{1} h_{2}}}\right.}\right)
$$

III. NEW EXACT SOLUTIONS OF RATIONAL EXPANSION FOR THE VARIABLE COEFFICIENT NON-LINEAR EQUA- TION WITH FORCED TERM

\section{A. Some new exact solutions of the Chaffee-Infante equation with variable coefficients}

Considering the Chaffee-Infante equation with variable coefficients:

$$
u_{t}-u_{x x}=\alpha(t) u\left(1-u^{2}\right)
$$

$\alpha(t)$ is arbitrary function of $t$ to be determined later.

By balancing the highest order partial derivative term and the nonlinear term in (16), we get the value of $m$,

$m=1$. According to the proposed method, we expand the solution of (16) in the form

$$
u(\xi)=a_{0}(t)+\frac{a_{1}(t) \psi(\xi)}{1+\mu \psi(\xi)}
$$

With the aid of Maple, substituting (17) along with (4) into (16), Then we get the following results:

$$
\begin{gathered}
k(t)=C_{0}, a_{0}(t)=0, l(t)=-2 \mu C_{0}{ }^{2} h_{1} t+C_{1}, \\
a_{1}(t)=C_{2} e^{\int \alpha d t}, \mu^{2}=-\frac{h_{1}}{h_{2}} .
\end{gathered}
$$

where $C_{0}, C_{1}, C_{2}$ are arbitrary constants, $\alpha(t)$ satisfy constraint relation as follow:

$$
-2 h_{2}{ }^{2} C_{0}{ }^{2}+2 h_{1}{ }^{2} C_{0}{ }^{2}+\alpha(t) C_{2}{ }^{2}\left(e^{\int \alpha d t}\right)^{2}=0
$$

From (17) , (18) , (19) and (5)-(15), we obtain the following solitonlike solutions for (16):

$$
\begin{aligned}
& u_{1}^{\prime}=\frac{C_{2} \sqrt{-h_{1} h_{2}} e^{\int \alpha d t} \tanh \left(\sqrt{-h_{1} h_{2}}(k x+l)+c_{1}\right)}{h_{2} \mu h_{1} \tanh \left(\sqrt{-h_{1} h_{2}}(k x+l)+c_{1}\right)} \\
& u_{2}^{\prime}=\frac{C_{2} \sqrt{-h_{1} h_{2}} e^{\int \alpha d t} \operatorname{coth}\left(\sqrt{-h_{1} h_{2}}(k x+l)+c_{2}\right)}{h_{2} \mu h_{1} \operatorname{coth}\left(\sqrt{-h_{1} h_{2}}(k x+l)+c_{2}\right)}
\end{aligned}
$$


$u_{3}^{\prime}=\frac{C_{2} \sqrt{-h_{1} h_{2}} e^{\int \alpha d t}\left(\operatorname{coth}\left(\sqrt{-4 h_{1} h_{2}}(k x+l)+c_{3}\right) \pm \csc \left(\sqrt{-4 h_{1} h_{2}}(k x+l)+c_{4}\right)\right)}{h_{2} \mu h_{1}\left(\operatorname{coth}\left(\sqrt{-4 h_{1} h_{2}}(k x+l)+c_{3}\right) \pm \csc \left(\sqrt{-4 h_{1} h_{2}}(k x+l)+c_{4}\right)\right)}$ $u_{4}^{\prime}=\frac{C_{2} \sqrt{-h_{1} h_{2}} e^{\int \alpha d t}\left(\tanh \left(\sqrt{-4 h_{1} h_{2}}(k x+l)+c_{5}\right) \pm i \operatorname{sech}\left(\sqrt{-4 h_{1} h_{2}}(k x+l)+c_{6}\right)\right)}{h_{2} \mu h_{1}\left(\tanh \left(\sqrt{-4 h_{1} h_{2}}(k x+l)+c_{5}\right) \pm i \operatorname{sech}\left(\sqrt{-4 h_{1} h_{2}}(k x+l)+c_{6}\right)\right)}$ we obtain the following index function form solutions for (16):

$$
u_{5}^{\prime}=\frac{C_{2} \sqrt{-h_{1} h_{2}} e^{\int \alpha d t}\left(1+c_{7} e^{2(k x+l) \sqrt{-h_{1} h_{2}}}\right)}{h_{2}\left(1-c_{7} e^{2(k x+l) \sqrt{-h_{1} h_{2}}}\right) \mu h_{1}\left(1+c_{7} e^{2(k x+l) \sqrt{-h_{1} h_{2}}}\right)}
$$

where $k(t)=C_{0}, l(t)=-2 \mu C_{0}{ }^{2} h_{1} t+C_{1}, c_{1}, c_{2}, c_{3}, c_{4}$, $c_{5}, C_{6}, c_{7}, C_{0}, C_{1}, C_{2}$ are arbitrary constants, and $\alpha(t)$ satisfy constraint relation Eq.(19).

B. Some new exact solutions of the KdV equation with variable coefficients

Considering the combined $\mathrm{KdV}$ equation with variable coefficients:

$$
u_{t}+\alpha(t) u u_{x}+m(t) u^{2} u_{x}+\beta(t) u_{x x x}=R(t)
$$

$\alpha(t), m(t), \beta(t), R(t)$ are arbitrary functions of $t$ to be determined later.

When $R(t)=0, \alpha(t), m(t), \beta(t)$ are constants, it turns to combined $\mathrm{KdV}$ equation, This equation is complex of the KdV and mKdV equations .

By balancing the highest order partial derivative term and the nonlinear term in (20), we get the value of $m$,

$m=1$. According to the proposed method, we expand the solution of (20) in the form

$$
u(\xi)=a_{0}(t)+\frac{a_{1}(t) \psi(\xi)}{1+\mu \psi(\xi)}
$$

where

$a_{i 0}=a_{i 0}(t), a_{i j}=a_{i j}(t)\left(i=1,2, \Lambda ; j=1,2, \Lambda, m_{i}\right)$, $\xi=\xi(x, t)=k(t) x+l(t), \quad \mu$ is constant, $\psi(\xi)$ satisfies Eq. (4).

With the aid of Maple, substituting (21) along with (4) into (20), Then we get the following results:

$k(t)=C_{0}, a_{0}(t)=0, \mu^{2}=\frac{h_{1} h_{2}}{3 h_{1}^{2}}, R(t)=R(t)$, $\beta(t)=\beta(t), \alpha(t)=\alpha(t)$,

$l(t)=\int \frac{-2 C_{0}^{3} \beta a_{1} h_{2} h_{1}^{2}-6 C_{0}^{3} \beta a_{1} \mu^{2} h_{1}^{3}+R}{a_{1} h_{1}} d t+C_{1}$

$a_{1}(t)=\int \frac{4 R \mu\left(\mu^{2} h_{1}-h_{2}\right)}{3 \mu^{2} h_{1}-h_{2}} d t+C_{2}$,

$m(t)=-\frac{1}{2} \frac{-4 R \mu^{5} h_{1}{ }^{2}-8 h_{2} R \mu^{3} h_{1}+3 C_{0} \alpha a_{1}{ }^{2} \mu^{4} h_{1}{ }^{3}+2 C_{0} \alpha a_{1}{ }^{2} \mu^{2} h_{1}{ }^{2} h_{2}-h_{2}{ }^{2} \alpha a_{1} h_{1} C_{0}-4 h_{2}{ }^{2} R \mu}{\mu C_{0} a_{1}{ }^{3} h_{1}^{3}\left(3 \mu^{2} h_{1}-h_{2}\right)}$ where $C_{0}, C_{1}, C_{2}$ are arbitrary constants, $a_{1}(t), R(t)$, $\beta(t)$ satisfy constraint relation as follow:

$$
\frac{\partial a_{1}}{\partial t}+\alpha a_{1}^{2} h_{1} C_{0}-2 R \mu-12 \beta \mu a_{1} h_{1}^{2} C_{0}^{3}\left(\mu^{2} h_{1}+h_{2}\right)=0
$$

From (21), (22), (23)and (5)-(15), we obtain the following solutions for (20):

1) From(21)-(23), when $h_{1} h_{2}>0$,

(a) From(5), (6) and (10), (11), we obtain the following triangle kind of periodic solutions of the variable coefficient $\mathrm{Kdv}$ equations

$u_{1}=\frac{\left(\int \frac{4 R \mu\left(\mu^{2} h_{1}-h_{2}\right)}{3 \mu^{2} h_{1}-h_{2}} d t+C_{2}\right) \frac{\sqrt{h_{1} h_{2}}}{h_{2}} \tan \left(\sqrt{h_{1} h_{2}}(k x+l)+c_{1}\right)}{1 \pm \frac{\sqrt{3}}{3} \tan \left(\sqrt{h_{1} h_{2}}(k x+l)+c_{1}\right)}$

$u_{2}=\frac{\left(\int \frac{4 R \mu\left(\mu^{2} h_{1}-h_{2}\right)}{3 \mu^{2} h_{1}-h_{2}} d t+C_{2}\right) \frac{\sqrt{h_{1} h_{2}}}{h_{2}} \cot \left(\sqrt{h_{1} h_{2}}(k x+l)+c_{2}\right)}{1 \pm \frac{\sqrt{3}}{3} \cot \left(\sqrt{h_{1} h_{2}}(k x+l)+c_{2}\right)}$

$u_{3}=\frac{\sqrt{h_{1} h_{2}}\left(\int \frac{4 R \mu\left(\mu^{2} h_{1}-h_{2}\right)}{3 \mu^{2} h_{1}-h_{2}} d t+C_{2}\right)\left[\csc \left(\sqrt{4 h_{1} h_{2}}(k x+l)+c_{3}\right) \pm \cot \left(\sqrt{4 h_{1} h_{2}}(k x+l)+c_{3}\right)\right]}{h_{2} \pm \frac{\sqrt{3}}{3} h_{2}\left[\csc \left(\sqrt{4 h_{1} h_{2}}(k x+l)+c_{3}\right) \pm \cot \left(\sqrt{4 h_{1} h_{2}}(k x+l)+c_{3}\right)\right]}$
$u_{4}=\frac{\sqrt{h_{1} h_{2}}\left(\int \frac{4 R \mu\left(\mu^{2} h_{1}-h_{2}\right)}{3 \mu^{2} h_{1}-h_{2}} d t+C_{2}\right)\left[\sec \left(\sqrt{4 h_{1} h_{2}}(k x+l)+c_{4}\right) \pm \tan \left(\sqrt{4 h_{1} h_{2}}(k x+l)+c_{4}\right)\right]}{h_{2} \pm \frac{\sqrt{3}}{3} h_{2}\left[\sec \left(\sqrt{4 h_{1} h_{2}}(k x+l)+c_{4}\right) \pm \tan \left(\sqrt{4 h_{1} h_{2}}(k x+l)+c_{4}\right)\right]}$

(b) From Eq.(14), we obtain the following index function form solutions of the variable coefficient Kdv equations.

$$
u_{5}=\frac{\sqrt{h_{1} h_{2}}\left(\int \frac{4 R \mu\left(\mu^{2} h_{1}-h_{2}\right)}{3 \mu^{2} h_{1}-h_{2}} d t+C_{2}\right)\left(1-i c_{5} e^{2 i(k x+l) \sqrt{h_{1} h_{2}}}\right)}{h_{2}\left(1+c_{5} e^{2 i(k x+l) \sqrt{h_{1} h_{2}}}\right) \pm \frac{\sqrt{3}}{3} h_{2}\left(1-i c_{5} e^{2 i(k x+l) \sqrt{h_{1} h_{2}}}\right)}
$$

where,

$$
l(t)=\int \frac{-2 C_{0}^{3} \beta a_{1} h_{2} h_{1}^{2}-6 C_{0}^{3} \beta a_{1} \mu^{2} h_{1}^{3}+R}{a_{1} h_{1}} d t+C_{1}
$$

$k(t)=C_{0}, C_{1}, C_{2}, C_{3}, C_{4}, C_{5}, C_{0}, C_{1}, C_{2}$ are arbitrary constants, and $a_{1}(t), R(t), \beta(t)$ satisfy constraint relation Eq.(23).

2) From Eq.(21)-(22), when $h_{1} h_{2}<0$,

(a) From (7),(8)and(12),(13), we obtain the following solitonlike solutions of the variable coefficient $\mathrm{Kdv}$ equations

$$
\begin{aligned}
& u_{6}=\frac{\left(\int \frac{4 R \mu\left(\mu^{2} h_{1}-h_{2}\right)}{3 \mu^{2} h_{1}-h_{2}} d t+C_{2}\right) \frac{\sqrt{-h_{1} h_{2}}}{h_{2}} \tanh \left(\sqrt{-h_{1} h_{2}}(k x+l)+c_{6}\right)}{1 \mu \frac{\sqrt{3}}{3} \tanh \left(\sqrt{-h_{1} h_{2}}(k x+l)+c_{6}\right)} \\
& u_{7}=\frac{\left(\int \frac{4 R \mu\left(\mu^{2} h_{1}-h_{2}\right)}{3 \mu^{2} h_{1}-h_{2}} d t+C_{2}\right) \frac{\sqrt{-h_{1} h_{2}}}{h_{2}} \tanh \left(\sqrt{-h_{1} h_{2}}(k x+l)+c_{6}\right)}{1 \mu \frac{\sqrt{3}}{3} \tanh \left(\sqrt{-h_{1} h_{2}}(k x+l)+c_{6}\right)} \\
& u_{8}=\frac{\sqrt{-h_{1} h_{2}}\left(\int \frac{4 R \mu\left(\mu^{2} h_{1}-h_{2}\right)}{3 \mu^{2} h_{1}-h_{2}} d t+C_{2}\right)\left[\operatorname{coth}\left(\sqrt{-4 h_{1} h_{2}}(k x+l)+c_{8}\right) \pm \operatorname{csch}\left(\sqrt{-4 h_{1} h_{2}}(k x+l)+c_{8}\right)\right]}{h_{2} \mu \frac{\sqrt{3}}{3} h_{2}\left[\operatorname{coth}\left(\sqrt{-4 h_{1} h_{2}}(k x+l)+c_{8}\right) \pm \operatorname{csch}\left(\sqrt{-4 h_{1} h_{2}}(k x+l)+c_{8}\right)\right]} \\
& u_{9}=\frac{\sqrt{-h_{1} h_{2}}\left(\int \frac{4 R \mu\left(\mu^{2} h_{1}-h_{2}\right)}{3 \mu^{2} h_{1}-h_{2}} d t+C_{2}\right)\left[\tanh \left(\sqrt{-4 h_{1} h_{2}}(k x+l)+c_{9}\right) \pm i \operatorname{sech}\left(\sqrt{-4 h_{1} h_{2}}(k x+l)+c_{9}\right)\right]}{h_{2} \mu \frac{\sqrt{3}}{3} h_{2}\left[\tanh \left(\sqrt{-4 h_{1} h_{2}}(k x+l)+c_{9}\right) \pm i \operatorname{sech}\left(\sqrt{-4 h_{1} h_{2}}(k x+l)+c_{9}\right)\right]}
\end{aligned}
$$


(b) From Eq.(15), we obtain the following index function form solutions of the variable coefficient $\mathrm{Kdv}$ equations.

$$
u_{10}=\frac{\sqrt{-h_{1} h_{2}}\left(\int \frac{4 R \mu\left(\mu^{2} h_{1}-h_{2}\right)}{3 \mu^{2} h_{1}-h_{2}} d t+C_{2}\right)\left(1+c_{10} e^{2(k x+l) \sqrt{-h_{1} h_{2}}}\right)}{h_{2}\left(1+c_{10} e^{2 i(k x+l) \sqrt{h_{1} h_{2}}}\right) \mu \frac{\sqrt{3}}{3} h_{2}\left(1-c_{10} e^{2(k x+l) \sqrt{-h_{1} h_{2}}}\right)} .
$$

where

$$
\begin{aligned}
& k(t)=C_{0}, \\
& l(t)=\int \frac{-2 C_{0}^{3} \beta a_{1} h_{2} h_{1}^{2}-6 C_{0}^{3} \beta a_{1} \mu^{2} h_{1}^{3}+R}{a_{1} h_{1}} d t+C_{1},
\end{aligned}
$$

$C_{1}, C_{2}, C_{3}, C_{4}, C_{5}, C_{0}, C_{1}, C_{2}$ are arbitrary constants, and $a_{1}(t), R(t), \beta(t)$ satisfy constraint relation Eq.(23).

Remark 3 Generalization: i) If we set the parameters $h_{1}$ and $h_{2}$ in (4) to different values, the ansatz in the tanh-function method [7], extended tanh-function method [8], modified extended tanh-function method [9], generalized hyperbolic-function method[10], the Riccati equation rational expansion method [11] and the generalized Riccati equation rational expansion [12] can all be recovered. That is to say, the ansäta proposed here, is more generalized. ii) In comparison to the constantcoefficient $\mathrm{KdV}$ equations in the document [3-10] , we remove some limitations for example $a_{i}(i=1,2$, $\Lambda m_{i}$ ) are constants and $\xi$ is linear function etc in the variable coefficient $\mathrm{KdV}$ equations, That is to say, it is more general in this paper.

Remark 4 Feasibility: In this paper, we reduce the restriction of unknowns, nature will increase the calculation complexity. We can detect complex tedious calculation by computer symbol system, but sometimes it is difficult, or even impossible. Therefore, for the unknown function we have to try to some special function to get the solution of differential equation.

\section{CONCLUSIONS}

In summary, based on the new Riccati equation rational expansion method, many generalized exact solutions of the variable coefficient combined $\mathrm{KdV}$ equation and Chaffee-Infante equation with forced term have been derived. More importantly, our method is powerful to find new solutions to various kinds of nonlinear evolution equations. We believe that this method should play an important role for finding exact solutions in the mathematical physics.

\section{ACKNOWLEDGMENT}

The work is supported by the National Nature Science Foundation of China (Grant No.41204035).

\section{REFERENCES}

[1] Ablowitz M J , Clarkson P A.Soliton, Nonlinear Evolution Equations and Inverse Scatting. New York: Cambridge University Press, 1991. http://dx.doi.org/10.1017/CBO9780511623998

[2] Chen Y, et al. Auto-Bäcklund transformation and exact solutions for modified nonlinear dispersive $\mathrm{mK}(\mathrm{m}, \mathrm{n})$ equations. Chaos, Solitions and Fractals, Vol. 17, Issue 2, 2003, pp. 693-698. http://dx.doi.org/10.1016/S0960-0779(02)00485-X

[3] Zuo Jinming.A computational method for the nonlinear BBM equation. Journal of Shandong University Natural Science, Vol. 68, Issue 4, 2010, pp. 47-50.

[4] Matveev V A, Salle M A. Darboux Transformations and Solitons. Springer-Verlag, 1991 http://dx.doi.org/10.1007/978-3662-00922-2

[5] Hu X B, Ma W X. Application of Hirota's bilinear formalism to the Toeplitz larrice-some special solitonlike solution. Phys. Lett A, Vol. 293, Issue 1, 2002, pp. 161-165. http://dx.doi.org/10.1016/ S0375-9601(01)00850-7

[6] Parjes E J, Duffy B R. Travelling Solitary Wave solutions to a compound Kdv-Burgers equation. Phys. lett A, Vol. 229, Issue 2, 2003, pp. 217-220.

[7] Fan E G. Extended tanh-function method and its applications to nonlinear equations. Phys. Lett A, Vol. 277, Issue 4, 2000, pp. 212-218. http://dx.doi.org/10.1016/S0375-9601(00)00725-8

[8] Gao Y T, Tian B. Generalized hyperbolic-function method with computerized symbolic computation to construct the solitonic solutions to nonlinear equations of mathematical physics. Comput Phys Comm, Vol. 145, Issue 1, 2010, pp. 158-164.

[9] Yan Z Y. New explicit travelling wave solutions for two new integrals coupled nonlinear evolution equations. Phys. Lett A, Vol. 292, Issue 4, 2001, pp. 100-106. http://dx.doi.org/10.1016/S03759601(01)00772-1

[10] Wang M L, Wan YM, Zhou Y B. An auto-Bäcklund transformation and exact solutions to a Generalized Kdv equation with variable coefficients and their applications. Phys Lett A, Vol. 313, Issue 5, 2009, pp. 45-51.

[11] Clarkson Peter A, Kruskal Martin D. New similarity reductions of the Boussinesq equation. Math Phys, Vol. 30, Issue 2, 2008, pp.2201-2213.

[12] Tang X Y, Lin J. Conditional similarity reductions of JimboMiwa equation via the classical Lie group approach. Commun Theor Phvs. Vol. 45, Issue 2, 2003, pp. 93-98.

[13] Lu D C, Hong B J. Explicit and exact solutions to the variable coefficient combined $\mathrm{KdV}$ equation with forced term, Phys. lett A, Vol. 55, Issue 11, 2009, pp. 5617-5622.

[14] Lu D C, Liu G L. A sub-ODE method for generalized Gardner and BBM equation with nonlinear terms of any order. Appl..Math.Comput. Vol. 35, Issue 2, 2010, pp. 217-220.

\section{AUTHORS}

F. Juan Liu is with the Department of Mathematics and Information, Henan Polytechnic University, CO 454000, China (e-mail: liujuan@ hpu.edu.cn).

S. Zhan-wei Hou is with the Department of Computer Science and Technology, Henan Polytechnic University, 454000, China (e-mail: hzw@ hpu.edu.cn).

This article is an extended and modified version of a paper presented at the International Conference on Mechanical Engineering, Automation and Material Science (MEAMS2012), held 22-23 December 2012, Wuhan, China. Received 09 April 2013. Published as resubmitted by the authors 01 May 2013. 